\title{
Novel Balanced Coupled-Line Bandpass Filters With Common-Mode Noise Suppression
}

\author{
Chung-Hwa Wu, Student Member, IEEE, Chi-Hsueh Wang, Member, IEEE, and Chun Hsiung Chen, Fellow, IEEE
}

\begin{abstract}
Novel balanced coupled-line bandpass filters, using suitable balanced coupled-line sections and quarter-wavelength resonators, are proposed. For design purposes, the differentialand common-mode equivalent half-circuits are established. Based on these circuits, a better balanced filter structure is implemented so that the desired differential-mode response may be realized and the level of common-mode noise may be minimized simultaneously. Besides, a suitable capacitive or inductive cross-coupled effect is introduced so as to create two transmission zeros for improving the filter selectivity; however, it also enhances the signal imbalance and degrades the common-mode rejection. In this study, various second- and fourth-order balanced filters are implemented to discuss the associated differential-mode responses and the signal-imbalance phenomena resulted from the cross-coupled effect. Specifically, the fourth-order filter with a common-mode rejection ratio of $40 \mathrm{~dB}$ within the passband is demonstrated and examined.
\end{abstract}

Index Terms-Balanced filter, common-mode noise suppression, coupled-line bandpass filter, quarter-wavelength resonator.

\section{INTRODUCTION}

B ALANCED circuits are important in building a modern communication system. Analog signals processed by a communication system are degraded by two different types of noises, namely, the environmental noise and device electronic noise. The former refers to the random disturbances that a circuit experiences through the dc power supply, ground lines, or substrate coupling. The latter includes the thermal noise, shot noise, and flicker noise, which come from the internal active device [1]. A noise limits the minimum signal level that a circuit can process with acceptable quality. The most important advantage of the balanced circuits with differential operation is the higher immunity to the environmental noise when compared with the unbalanced circuits with single-ended signaling.

Over the past few years, several balanced circuit topologies have been developed to connect with the monopole antenna for which a balun is needed to convert a balanced signal into an unbalanced one. Typical balun configurations reported include those based on distributed [2], [3], lumped [4], or lumped-distributed elements [5]-[7]. Some balun topologies offering bandpass-type transmission characteristics were also discussed in [8] and [9]. After that, several single-to-differential bandpass filters

Manuscript received October 12, 2006; revised October 26, 2006. This work was supported by the National Science Council of Taiwan, R.O.C., under Grant NSC 95-2752-E-002-001-PAE, Grant NSC 95-2219-E-002-008, and Grant NSC 95-2221-E-002-196.

The authors are with the Department of Electrical Engineering and Graduate Institute of Communication Engineering, National Taiwan University, Taipei 106, Taiwan, R.O.C. (e-mail: chchen@ew.ee.ntu.edu.tw).

Digital Object Identifier 10.1109/TMTT.2006.889147 were proposed to replace the conventional balun together with a bandpass filter. In [10], a second-order combline filter in series with the conventional Marchand balun was realized in the low-temperature co-fired ceramic (LTCC) substrate. Based on the same concept, a modified version was presented in [11]. In [12], by introducing a capacitive loading, an additional transmission zero was created to optimize the filter response. Recently, an LTCC circuit architecture that combines the functionalities of balun and bandpass filter was proposed in [13]. Owing to the adoption of lumped elements, the realization of the balanced-to-unbalanced filter in [13] is not easy in a higher microwave region.

Traditional off-chip radiating elements suffer from additional interconnect losses and cannot be duplicated like the on-chip antennas. The small wavelength at millimeter-wave frequency and the mature CMOS fabrication technology make it possible to use on-chip metal layers to fabricate small integrated antennas [14], [15]. Although still faced with a problem of improving the antenna efficiency, there will be more and more integrated circuits implemented in the fully differential form under the trend of system-on-chip (SOC). The on-chip integrated antenna usually takes the form of a dipole, which needs a balanced filter for differential operation. A well-designed differential-to-differential balanced bandpass filter should exhibit the desired bandpass frequency response in differential operation and should be able to reduce the level of the common-mode signal so as to increase the signal-to-noise-ratio in the receiver and to improve the efficiency of the dipole antenna in the transmitter.

Pervious studies on differential-to-differential balanced filter designs are rather limited. In [16], a 40-GHz balanced filter was proposed based on a half-wavelength $(\lambda / 2)$ patch resonator and four quarter-wavelength $(\lambda / 4)$ transmission lines. That filter structure presents a good common-mode rejection ability, but it has the drawback of bulky size. Recently, a 2-GHz balanced filter [17] was realized based on conventional parallel-coupledline structures [18]-[22]. Compared with [16], the coupled-line balanced filter in [17] exhibits the advantages of a compact size and simple synthesis procedure; however, it shows a poor common-mode rejection ability, which is crucial in the balanced filter design.

In this study, novel balanced coupled-line structures are proposed to implement the balanced filters so as to give an excellent common-mode rejection in addition to providing the desired differential-mode bandpass response. The desired differentialand common-mode responses are realized by a suitable choice of balanced coupled-line sections. Specifically, by properly designing the circuits associated with the common-mode signal, a very low common-mode signal level around $f_{0}^{d}$ (the center frequency of the differential-mode response) is achieved without any degradation of insertion loss in differential-mode operation. 


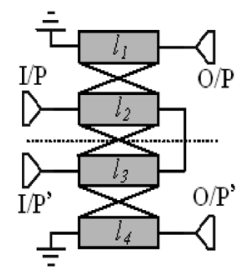

(a)

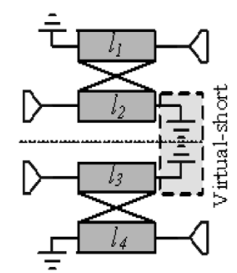

(b)

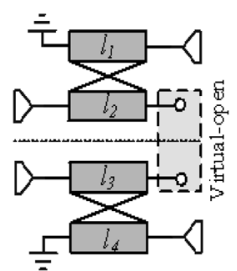

(c)
Fig. 1. Type-I balanced coupled-line section. (a) Circuit structure. (b) Differential-mode equivalent circuit. (c) Common-mode equivalent circuit.

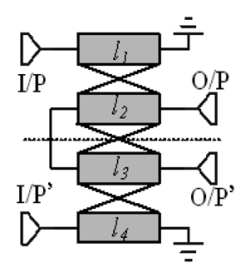

(a)

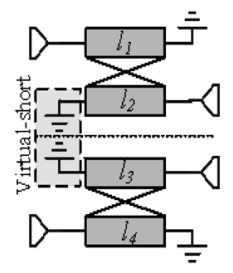

(b)

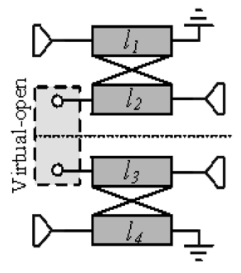

(c)
Fig. 2. Type-II balanced coupled-line section. (a) Circuit structure. (b) Differential-mode equivalent circuit. (c) Common-mode equivalent circuit.

For design purposes, the equivalent half-circuits are also established so that both differential- and common-mode frequency responses may be discussed separately. In this study, two types of second-order balanced filters are realized using the microstrip structure. To further improve the filter response, two types of fourth-order balanced filters are realized based on the novel dual-metal-plane structure consisting of both a microstrip and a coplanar waveguide. The proposed balanced filter circuits are implemented, measured, and carefully examined.

\section{BAlanced Coupled-Line SEctions}

A realization of the structure, which gives both desired differential-mode bandpass response and minimum common-mode noise level, is essential in developing a balanced filter. Figs. 1(a) and 2(a) show two types (types I and II) of balanced coupledline sections, which are capable of realizing such desired responses. Among these two structures, the most noticeable difference is in the positions of input/output ports and shorted ends. In Fig. 1(a), the differential input ports are through the elements $l_{2}$ and $l_{3}$ of the balanced coupled-line section, and the differential output ports are through the elements $l_{1}$ and $l_{4}$. Alternatively, in Fig. 2(a), the differential input ports are through the elements $l_{1}$ and $l_{4}$, and the differential output ports are through the elements $l_{2}$ and $l_{3}$. As to the locations of short ends, they are on the left-hand side of Fig. 1(a), whereas they are on the right-hand side of Fig. 2(a).

For balanced operation, the balanced coupled-line sections may be decomposed into two equivalent circuits under odd- and even-mode excitations. Under odd-mode excitation, although the structures in Figs. 1(a) and 2(a) are quite different in circuit layouts, they can both be represented by the same equivalent circuits, as shown in Figs. 1(b) and 2(b). However, under evenmode excitation, the structures in Figs. 1(a) and 2(a) should be represented by the two different equivalent circuits shown in Figs. 1(c) and 2(c), respectively.
Being composed of a quarter-wavelength $(\lambda / 4)$ coupled-line section with its two ends shorted, the odd-mode equivalent circuit shown in Fig. 1(b) or 2(b) exhibits a bandpass frequency response with the center frequency at $f_{0}$. On the other hand, the even-mode equivalent circuit shown in Fig. 1(c) or 2(c) would present the all-stop frequency response at $f_{0}$ because it consists of a $\lambda / 4$ coupled-line section with one end shorted and the other end opened.

Theoretically, Figs. 1(c) and (2c) with ideal ground both present the all-stop characteristics under common-mode operation. However, in the practical case, the shorted-ends of coupled lines will be realized by the nonideal via-holes, which may result in different behaviors in the common-mode operation. To minimize the common-mode noise, the coupled-line sections should properly be chosen.

\section{SECOND-ORDER FILTERS}

A well-designed balanced bandpass filter should possess the capability of reducing the level of common-mode noise in addition to providing the desired bandpass frequency response in differential-mode operation. In [17], only the differential-mode operation was discussed without considering the common-mode response. Actually, both differential- and common-mode responses should be taken into consideration so as to give a good common-mode rejection. Based on the balanced coupled-line structures shown in Figs. 1(a) and 2(a), two types of second-order balanced filters may be developed to give a good common-mode rejection ability. Fig. 3 shows the layouts of proposed second-order balanced filters (types I and II), and their corresponding differential- and common-mode equivalent half-circuits are shown in Figs. 4 and 5. Specifically, by decomposing the balanced filter structures into two different equivalent half-circuits under odd- and even-mode excitations, one may discuss the differential- and common-mode responses accordingly.

The proposed balanced filters (Fig. 3) are simulated by the commercial software Sonnet and AWR Microwave Office (MWO). All the circuits are fabricated on the FR4 substrate $\left(\varepsilon_{r}=4.3, \tan \delta=0.02\right.$, and the thickness $\left.=1 \mathrm{~mm}\right)$. The balanced filter, as a four-port device, is first measured by Agilent's E5071B network analyzer to give the standard four-port $S$-parameters $S^{\text {std }}$. The two-port differential- and common-mode $S$-parameters $S^{d d}$ and $S^{c c}$ may then be extracted from the four-port $S$-parameters $S^{\text {std }}$, as given by [23]

$$
\begin{aligned}
S^{m m} & =M S^{\text {std }} M^{-1} \\
M & =\frac{1}{\sqrt{2}}\left[\begin{array}{cccc}
1 & -1 & 0 & 0 \\
0 & 0 & 1 & -1 \\
1 & 1 & 0 & 0 \\
0 & 0 & 1 & 1
\end{array}\right] .
\end{aligned}
$$

Basically, the mixed-mode $S$-parameters $\left(S^{m m}\right)$ may be decomposed into

$$
S^{m m}=\left[\begin{array}{ll}
S^{d d} & S^{d c} \\
S^{c d} & S^{c c}
\end{array}\right]
$$

in which

$$
S^{c c}=\left[\begin{array}{ll}
S_{11}^{c c} & S_{12}^{c c} \\
S_{21}^{c c} & S_{22}^{c c}
\end{array}\right]
$$




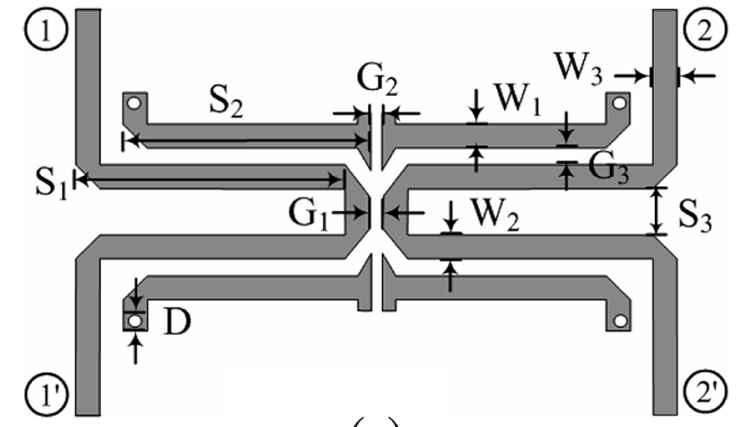

(a)

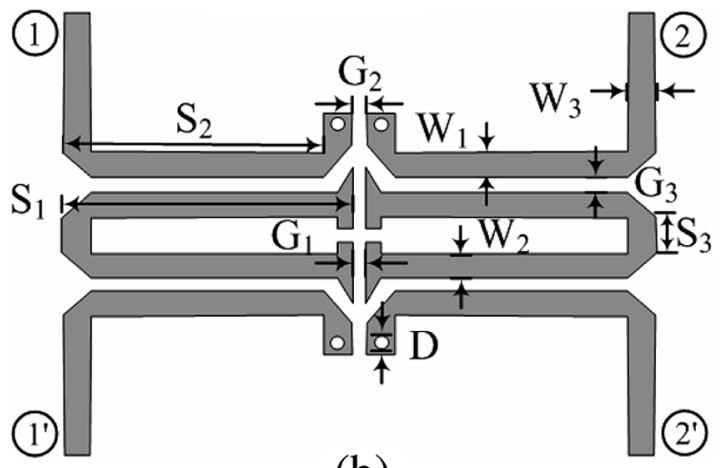

(b)

Fig. 3. Physical layouts of the proposed second-order balanced filters. (a) Type I $S_{1}=20.1 \mathrm{~mm}, S_{2}=17.5 \mathrm{~mm}, L_{3}=4 \mathrm{~mm}, W_{1}=1.9 \mathrm{~mm}, W_{2}=$ $1.9 \mathrm{~mm}, W_{3}=1.7 \mathrm{~mm}, G_{1}=0.5 \mathrm{~mm}, G_{2}=0.3 \mathrm{~mm}, G_{3}=0.3 \mathrm{~mm}$, $D=1 \mathrm{~mm})$. (b) Type II $\left(S_{1}=17.8 \mathrm{~mm}, S_{2}=16.8 \mathrm{~mm}, S_{3}=2.7 \mathrm{~mm}\right.$, $W_{1}=1.9 \mathrm{~mm}, W_{2}=1.9 \mathrm{~mm}, W_{3}=1.7 \mathrm{~mm}, G_{1}=0.3 \mathrm{~mm}, G_{2}=$ $0.5 \mathrm{~mm}, G_{3}=0.3 \mathrm{~mm}, D=1 \mathrm{~mm}$ ).

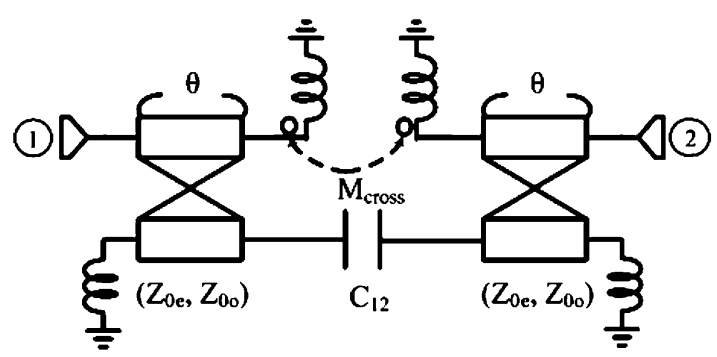

(a)

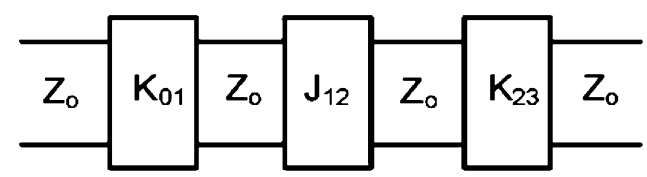

(b)

Fig. 4. Differential-mode equivalent half-circuit of the proposed second-order balanced filters in Fig. 3. (a) $M_{\text {cross }} \neq 0$. (b) $M_{\text {cross }}=0$.

$$
S^{d d}=\left[\begin{array}{ll}
S_{11}^{d d} & S_{12}^{d d} \\
S_{21}^{d d} & S_{22}^{d d}
\end{array}\right]
$$

represent the two-port differential- and common-mode $S$-parameters, respectively. Similar expressions may be used for the two-port cross-mode $S$-parameters $S^{d c}$ and $S^{c d}$. For an ideal balanced structure, the cross-mode $S$-parameters $S^{d c}$ and $S^{c d}$ would be a two-by-two matrix of zero elements.

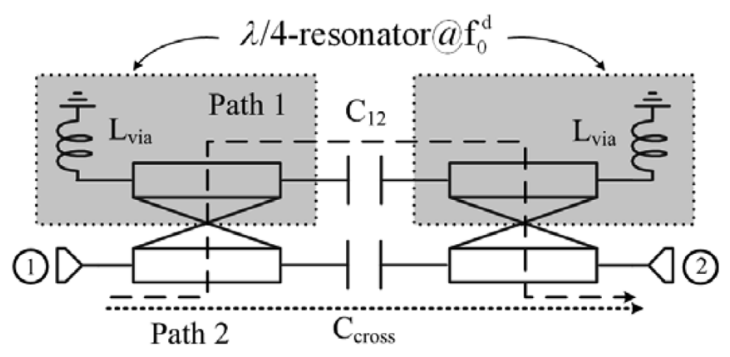

(a)

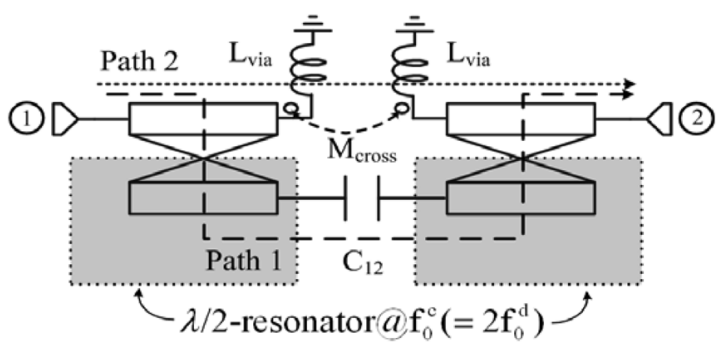

(b)

Fig. 5. Common-mode equivalent half-circuits of the proposed second-order balanced filters in Fig. 3. (a) Type I. (b) Type II.

\section{A. Differential-Mode Response}

For the balanced filters shown in Fig. 3, the corresponding differential-mode equivalent half-circuit [shown in Fig. 4(a)] is composed of two bandpass-response coupled-line sections [shown in Figs. 1(b) or (2b)], which are connected by a series capacitor $C_{12}$. This capacitor $C_{12}$ is realized by the open-ended edge coupled structure associated with the gap $G_{2}$ in Fig. 3(a) or the gap $G_{1}$ in Fig. 3(b). In addition, a mutual inductance $M_{\text {cross }}$ is introduced to provide a cross-coupled path for creating two transmission zeros. As shown in Fig. 3, the cross-coupled inductance $M_{\text {cross }}$ for each type is achieved with different physical structures. In the type-I filter, due to the virtual short in the center line, the inductive cross-coupling $M_{\text {cross }}$ is realized along the coupled-line sections associated with the gap $G_{1}$ in Fig. 3(a). On the other hand, the inductive cross-coupling is formed between two via-holes associated with the gap $G_{2}$ in Fig. 3(b). Physically, the location of the transmission zeros may simply be adjusted by controlling the mutual inductance $M_{\text {cross }}$ through varying the gapwidth $G_{1}$ [of Fig. 3(a)] or $G_{2}$ [of Fig. 3(b)].

With the cross-coupled inductance neglected $\left(M_{\text {cross }}=0\right)$, the differential-mode equivalent half-circuit Fig. 4(a) for the second-order filters composed of $\lambda / 4$ resonators may further be represented by the one in Fig. 4(b). The design procedures for the filter in Fig. 4(b) are well documented in [21] and [22]. For the differential-mode operation, the second-order filter is designed with a center frequency $f_{0}^{d}=f_{0}=2 \mathrm{GHz}$, a 3-dB bandwidth of $10 \%$, and a characteristic impedance $Z_{0}=50 \Omega$ based on the maximally flat response. The corresponding circuit parameters are obtained as follows:

$$
\begin{aligned}
\left(Z_{0 e}, Z_{0 o}\right) & =(64.56 \Omega, 40.99 \Omega) \\
\theta & =86.8^{\circ} \text { at } 2 \mathrm{GHz} \\
C_{12} & =0.088 \mathrm{pF} .
\end{aligned}
$$

Based on these parameters, the type-I and type-II filters are fabricated on the FR4 substrate with the layouts shown in 


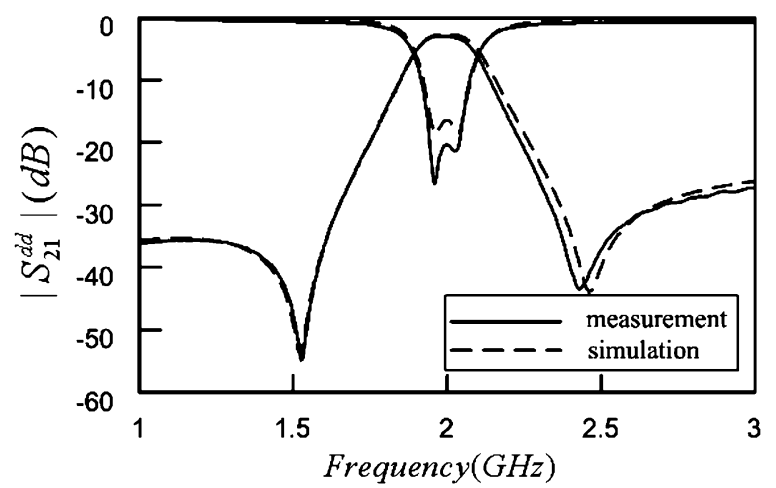

(a)

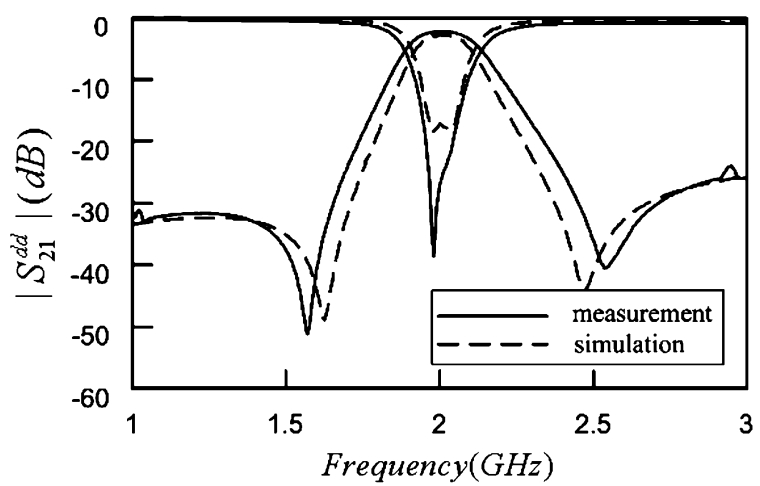

(b)

Fig. 6. Measured and simulated differential-mode responses of the proposed second-order filters in Fig. 3. (a) Type I. (b) Type II.

Fig. 3(a) and (b). These filters are simulated using the full-wave simulator Sonnet, and are measured using the four- to two-port conversion technique, as given by (1)-(5). The narrowband measured and simulated differential-mode responses are shown in Fig. 6. Good agreement between measured and simulated results is observed with only a slight deviation in bandwidth. For the type-I filter, the measured center frequency is at $2 \mathrm{GHz}$, with a minimum differential-mode insertion loss of $2.9 \mathrm{~dB}$ and a 3-dB bandwidth of $10.5 \%$. For the type-II filter, the measured center frequency is at $2 \mathrm{GHz}$ with a minimum differential-mode insertion loss of $2.3 \mathrm{~dB}$ and a $3-\mathrm{dB}$ bandwidth of $12 \%$.

\section{B. Common-Mode Response}

For the second-order balanced filter structures shown in Fig. 3(a) and (b), the corresponding common-mode equivalent half-circuits are illustrated in Fig. 5(a) and (b), respectively. By neglecting the loaded inductances $L_{\text {via }}$ associated with the via-holes and the cross-coupled effect due to $M_{\text {cross }}$ or $C_{\text {cross }}$, the two equivalent half-circuits would exhibit the all-stop frequency responses in common-mode operation since these circuits are mainly composed of the all-stop coupled-line sections, as shown in Figs. 1(c) and 2(c). The existence of via-hole inductances and cross-coupled effect destroys the all-stop characteristic and causes the unwanted signal-imbalance $\left(\left|S_{21}\right| \neq\left|S_{2^{\prime} 1}\right|\right.$ and $\left.\angle S_{21}-\angle S_{2^{\prime} 1} \neq 180^{\circ}\right)$ around $f_{0}^{d}$ (the passband center frequency of differential-mode operation).

To demonstrate the effects of the loaded inductors $L_{\text {via }}$ and the cross-coupled path (path 2), the common-mode insertion

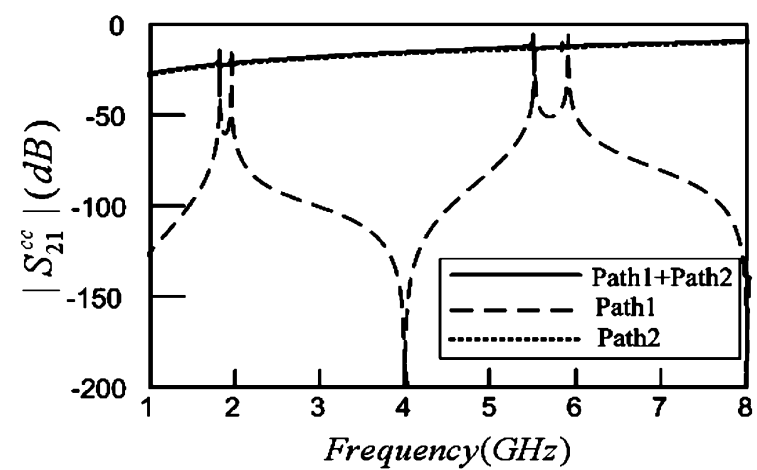

(a)

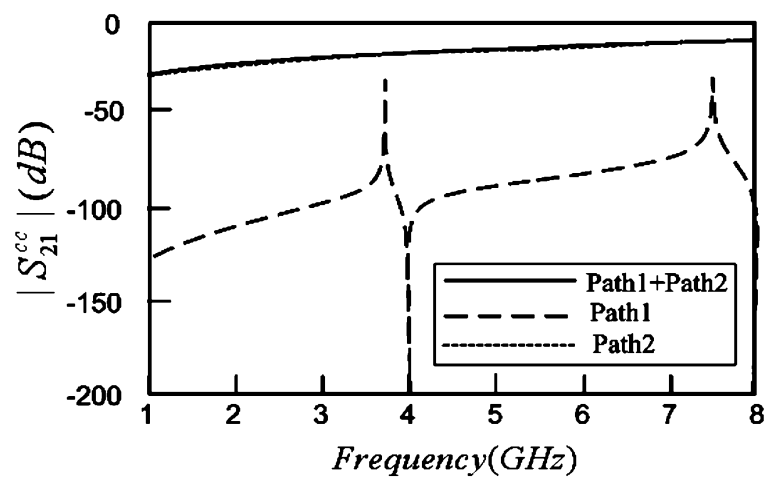

(b)

Fig. 7. Simulated common-mode responses of the signals along paths 1 and 2 of the circuit models shown in Fig. 5 with $L_{\text {via }}=0.1 \mathrm{nH}$. (a) Type I. (b) Type II.

losses along two signal paths are simulated by the MWO circuit simulator and are shown in Fig. 7(a) and (b). Here, path 1 represents the main signal path primarily composed of two all-stop coupled-line sections. Also included in path 1 is the series capacitor $C_{12}$ alone, as in Fig. 5(b), or the capacitor $C_{12}$ plus the loaded inductors $L_{\text {via }}$, as in Fig. 5(a). Path 2 denotes the cross-coupled path through the cross-coupled capacitance $C_{\text {cross }}$ [see Fig. 5(a)] or the cross-coupled mutual inductance $M_{\text {cross }}$ [see Fig. 5(b)]. Fig. 7 indicates that the common-mode response is mainly determined by the signal through path 2 ; however, its response around $f_{0}^{d}(=2 \mathrm{GHz})$ is largely influenced by the signal through path 1 .

For the type-I filter [see Fig. 3(a)], the resonator under the common-mode operation is composed of a $\lambda / 4$ transmission line section with one end shorted and another end opened; here, $\lambda$ is the guided wavelength corresponding to the frequency $f_{0}^{d}=$ $2 \mathrm{GHz}$. Therefore, the center frequency $f_{0}^{c}$ of the common-mode response of the type-I filter would be the same as that of the differential-mode response $\left(f_{0}^{c}=f_{0}^{d}\right)$. Besides, the signal along path 1 presents two peaks around $f_{0}^{d}\left(=f_{0}^{c}\right)$, which produce two spurs in the resultant common-mode response around $f_{0}^{d}$, thereby degrading the common-mode rejection ability.

For the type-II filter, the resonator under common-mode operation behaves as a $\lambda / 2$ open-end resonator, where $\lambda$ is the guided wavelength corresponding to the frequency $f_{0}^{c}=2 f_{0}^{d}=4 \mathrm{GHz}$. Therefore, the central frequency $f_{0}^{c}$ of the common-mode response would be twice the differential-mode response $\left(f_{0}^{c}=\right.$ $2 f_{0}^{d}$ ). Although the signal along path 2 also shows a peak around $f_{0}^{c}$, its contribution to the resultant common-mode response is negligible, as depicted in Fig. 7(b). 


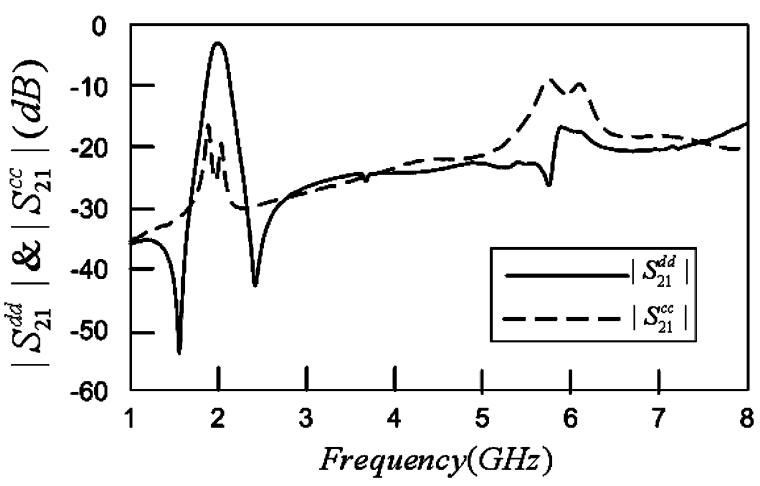

(a)

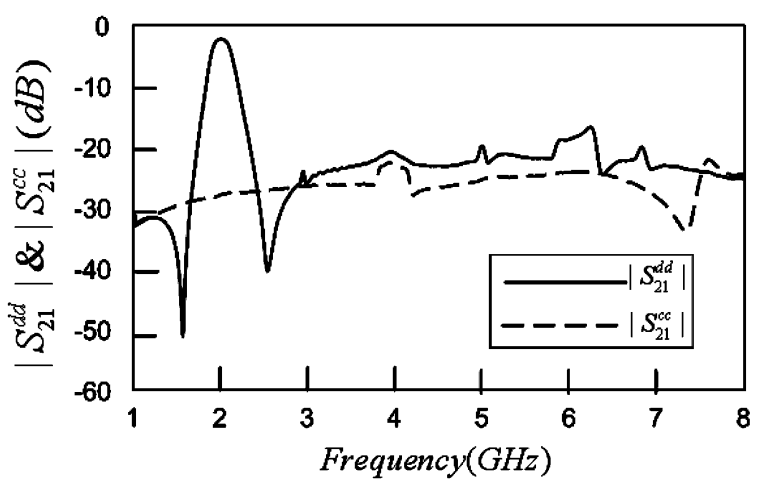

(b)

Fig. 8. Measured differential- and common-mode frequency responses of the proposed second-order filters in Fig. 3. (a) Type I. (b) Type II.

The existence of loaded inductors $L_{\text {via }}$ is unavoidable in practice realization. However, the type-II design may push the center frequency $f_{0}^{c}$ of the common-mode response to the higher frequency band $\left(f_{0}^{c}=2 f_{0}^{d}\right)$, thereby improving the common-mode rejection ability.

The wideband differential- and common-mode measured responses are shown in Fig. 8(a) and (b) for comparison. In the type-I design, the minimum common-mode insertion loss is $-16.8 \mathrm{~dB}$ around $f_{0}^{d}$; while in type-II design, it is $-28 \mathrm{~dB}$ around $f_{0}^{d}$, and all below $-25 \mathrm{~dB}$ from 0.5 to $8.5 \mathrm{GHz}$. As a result, an improvement of $11.2 \mathrm{~dB}$ in common-mode noise rejection around $f_{0}^{d}$ could be achieved by using the type-II structure.

\section{FOURTH-ORDER FILTERS}

Intuitively, the rejection level of the common-mode signal can further be suppressed by increasing the filter order. A fourthorder balanced filter may be achieved by introducing two additional series capacitors at the input/output ends of the proposed filter structures in Fig. 3. Figs. 9 and 10 show the layouts of the proposed fourth-order filters. The corresponding differential- and common-mode equivalent half-circuits are also shown in Figs. 11 and 12. Specially, the series input/output capacitors $C_{01}$, to realize the $J$-inverter, are implemented by the metal-insulator-metal (MIM) structure so as to achieve a higher capacitance value.

As shown in Figs. 9 and 10, the proposed fourth-order balanced filters are constructed using the dual-metal-plane structures. Here, the microstrip coupled-line sections are lo-

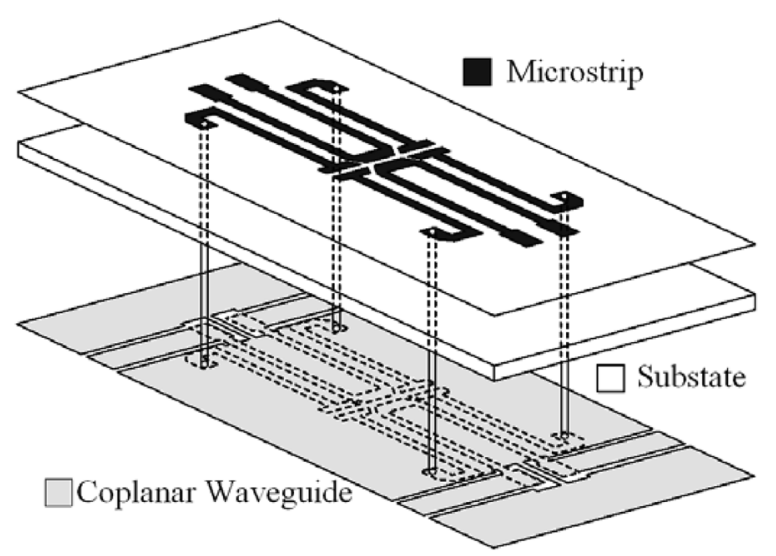

(a)

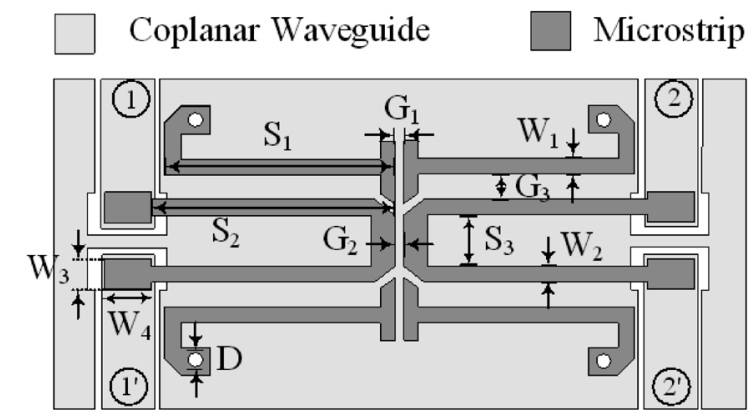

(b)

Fig. 9. (a) 3-D physical layout and (b) top-/bottom-plane layout of the proposed fourth-order type-I balanced filter $\left(S_{1}=15.1 \mathrm{~mm}, S_{2}=17.7 \mathrm{~mm}, S_{3}=\right.$ $3 \mathrm{~mm}, W_{1}=1.1 \mathrm{~mm}, W_{2}=1.1 \mathrm{~mm}, W_{3}=2.1 \mathrm{~mm}, W_{4}=3.2 \mathrm{~mm}$, $G_{1}=0.5 \mathrm{~mm}, G_{2}=0.3 \mathrm{~mm}, G_{3}=1.8 \mathrm{~mm}, D=1 \mathrm{~mm}$ ).

\section{Coplanar Waveguide $\square$ Microstrip}

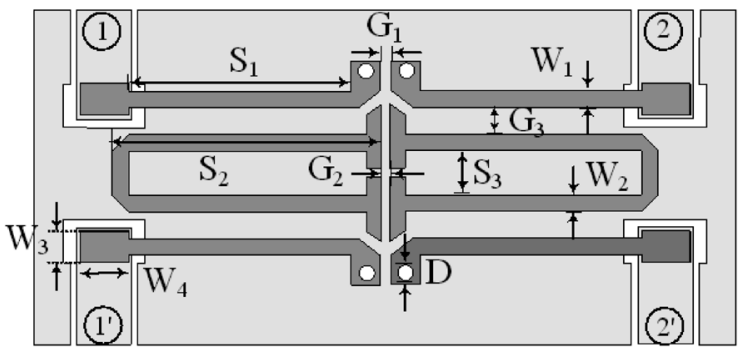

Fig. 10. Top-/bottom-plane layout of the proposed fourth-order type-II balanced filter $\left(S_{1}=15.7 \mathrm{~mm}, S_{2}=16.6 \mathrm{~mm}, S_{3}=3.5 \mathrm{~mm}, W_{1}=1.1 \mathrm{~mm}\right.$, $W_{2}=1.1 \mathrm{~mm}, W_{3}=2.1 \mathrm{~mm}, W_{4}=3.2 \mathrm{~mm}, G_{1}=0.3 \mathrm{~mm}, G_{2}=$ $0.3 \mathrm{~mm}, G_{3}=1.7 \mathrm{~mm}, D=1 \mathrm{~mm}$ ).

cated on the top metal plane, and the coplanar waveguide (CPW) input/output transmission lines together with the ground plane of the microstrip are on the bottom metal plane. The MIM capacitor is achieved between the CPW input/output transmission line on the bottom metal plane and the microstrip coupled-line section on the top metal plane.

\section{A. Differential-Mode Response}

Fig. 11 demonstrates the odd-mode equivalent half-circuit of the fourth-order balanced filters. The design of the filter under odd-mode operation may follow the similar procedures, as mentioned in Section III. These filters are designed with $f_{0}^{d}=f_{0}=$ 


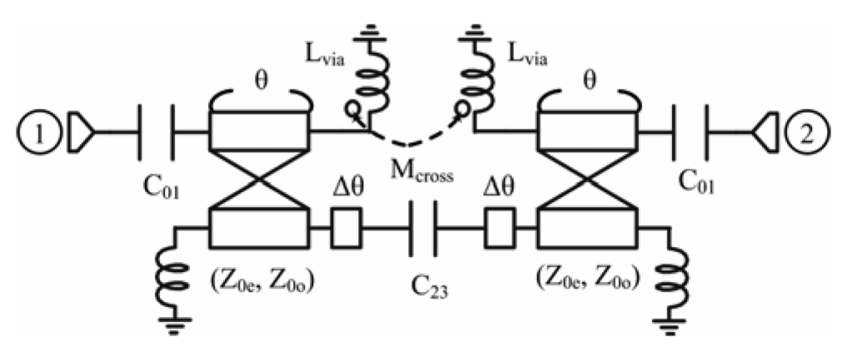

Fig. 11. Differential-mode equivalent half-circuit of fourth-order balanced filters in Figs. 9 and 10.

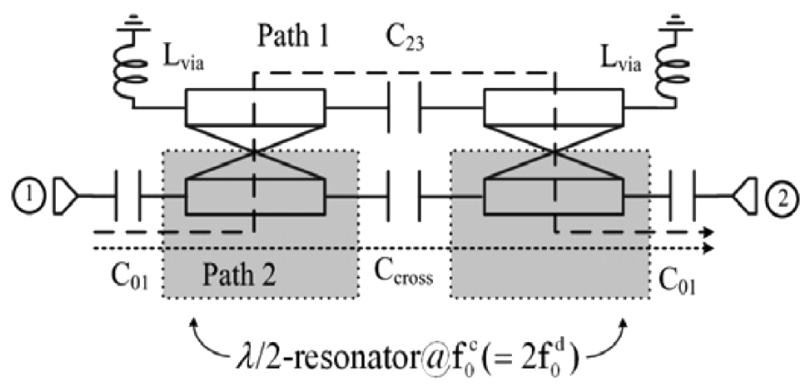

(a)

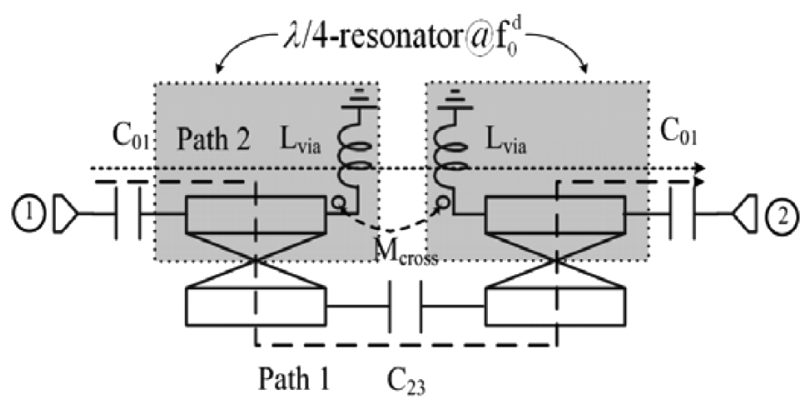

(b)

Fig. 12. Common-mode equivalent half-circuits of fourth-order balanced filters in Figs. 9 and 10. (a) Type-I. (b) Type-II.

$2 \mathrm{GHz}, 3-\mathrm{dB}$ bandwidth $=10 \%$, and $Z_{0}=50 \Omega$. The corresponding circuit parameters are obtained based on the fourthorder maximally flat response as follows:

$$
\begin{aligned}
\left(Z_{0 e}, Z_{0 o}\right) & =(53.52 \Omega, 46.92 \Omega) \\
\theta & =72.24^{\circ} \text { at } 2 \mathrm{GHz} \\
\Delta \theta & =15.33^{\circ} \text { at } 2 \mathrm{GHz} \\
C_{01} & =0.568 \mathrm{pF} \\
C_{23} & =0.0678 \mathrm{pF} .
\end{aligned}
$$

For the fourth-order filters shown in Figs. 9 and 10, the cross-coupled effect is also introduced between input/output resonators so as to enhance the filter selectivity. The wideband differential-mode measured responses are shown in Fig. 13. In the type-I design, the measured $f_{0}^{d}$ is at $1.99 \mathrm{GHz}$ with a minimum differential-mode insertion loss of $3.73 \mathrm{~dB}$ and a 3 -dB bandwidth of $11 \%$. For the type-II filter, the measured $f_{0}^{d}$ is at $2.05 \mathrm{GHz}$ with a minimum differential-mode insertion loss of 3.98- and a 3-dB bandwidth of $9.7 \%$.

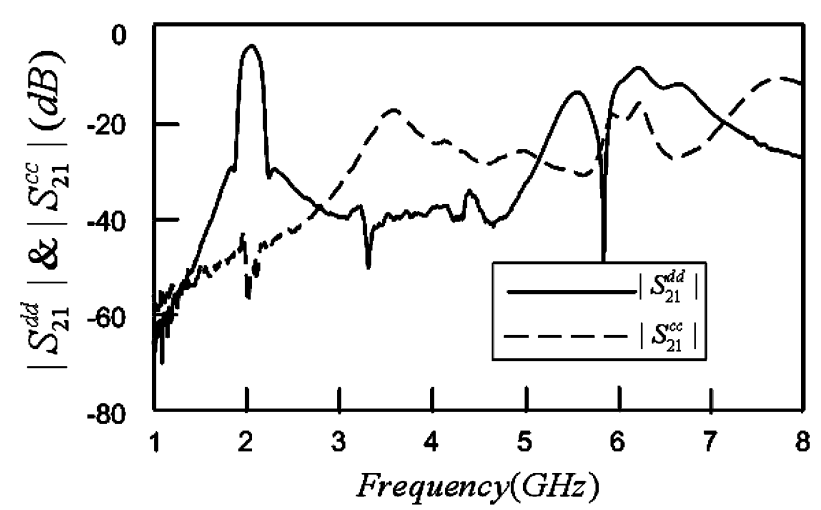

(a)

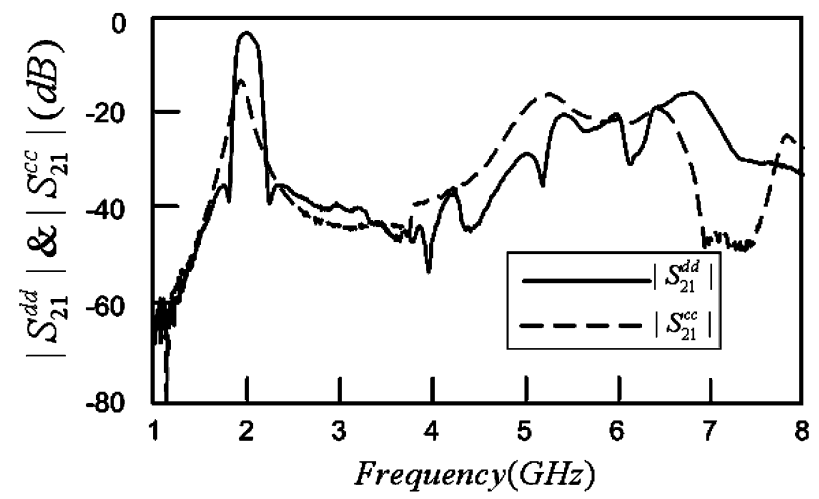

(b)

Fig. 13. Measured differential- and common-mode frequency responses of the proposed fourth-order filters. (a) Type I. (b) Type II.

\section{B. Common-Mode Response}

Shown in Fig. 12 are the corresponding even-mode equivalent half-circuits of the fourth-order balanced filters. Based on the same concept as mentioned in Section III, two signal paths are introduced to discuss the common-mode responses, which are shown in Fig. 14. Paying attention to the response for path 1 in Fig. 14, it is found that the frequency response of each type along path 1 is similar to that of the second-order one. However, having benefited from the higher order structure, the level of the common-mode signal along path 1 has been further suppressed below $-50 \mathrm{~dB}$ so that its contribution to the resultant commonmode response is of minor significance.

The common-mode responses of fourth-order filters (Figs. 9 and 10) are mainly dominated by the signal along the cross-coupled path (path 2). The even-mode equivalent half-circuits in Fig. 12 are useful in discussing this signal along path 2 . Note that the type-II filter is primarily composed of $\lambda / 4$ resonators with one end shorted and another end opened [see Fig. 12(b)], making its resonant frequency $f_{0}^{c}$ equal to $f_{0}^{d}(\sim 2 \mathrm{GHz})$. Thus, both differential- and common-mode responses would have the same passband around $f_{0}^{d}\left(=f_{0}^{c}\right)$. This explains why the type-II filter has a poor common-mode rejection around $f_{0}^{d}$. Alternatively, the type-I filter is based on the $\lambda / 2$ open-end transmission line resonator, which would push the passband center frequency $f_{0}^{c}$ up to $2 f_{0}^{d}(\sim 4 \mathrm{GHz})$, as shown in Fig. 14(a). Therefore, the type-I design (Fig. 9) would achieve better common-mode rejection around $f_{0}^{d}$. 


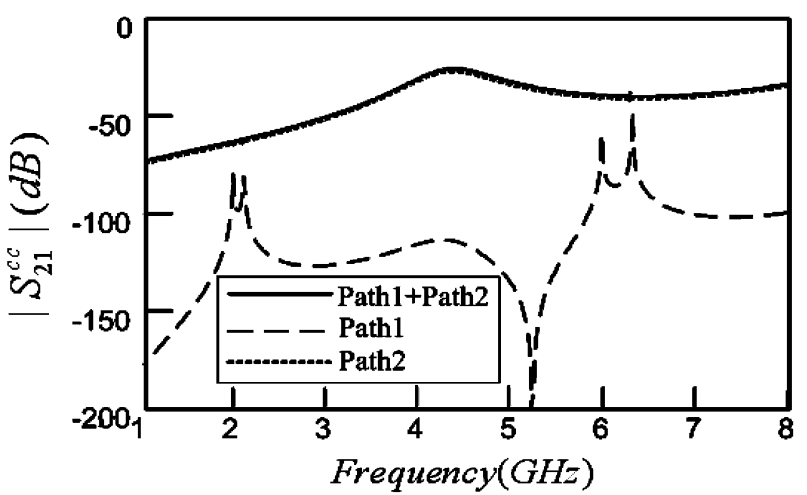

(a)

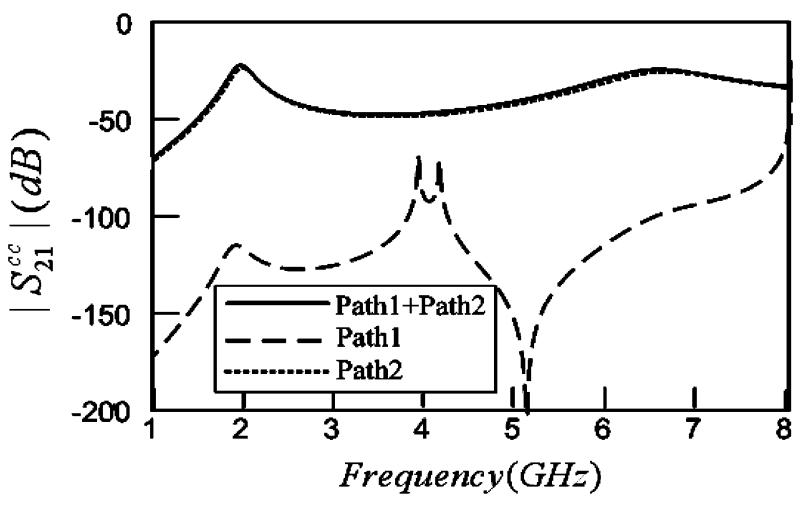

(b)

Fig. 14. Frequency responses of the two signal paths with $L_{\text {via }}=0.1 \mathrm{nH}$ for fourth-order design. (a) Type I. (b) Type II.

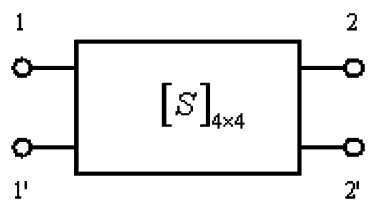

Fig. 15. Practical balanced circuit.

The wideband common-mode measured responses are also shown in Fig. 13. The minimum common-mode insertion loss is $42 \mathrm{~dB}$ at $1.97 \mathrm{GHz}$ in the type-I design, and minimum commonmode insertion loss is $15 \mathrm{~dB}$ at $1.95 \mathrm{GHz}$ in the type-II design.

\section{COMmon-Mode ReJECTION RATIO (CMRR)}

To provide a simple figure-of-merit for characterizing the implement balanced filters, the CMRR defined by

$$
\mathrm{CMRR}=20 \log \frac{\left|S_{21}^{d d}\right|}{\left|S_{21}^{c c}\right|}(\mathrm{dB})
$$

is adopted in this study. Basically, the value of the CMRR may be used to quantitatively discuss the degree of resemblance between the implemented balanced filter and the ideal balanced one.

For the well-designed balanced circuit (Fig. 15) to realize an ideal balanced one, the corresponding differential- and

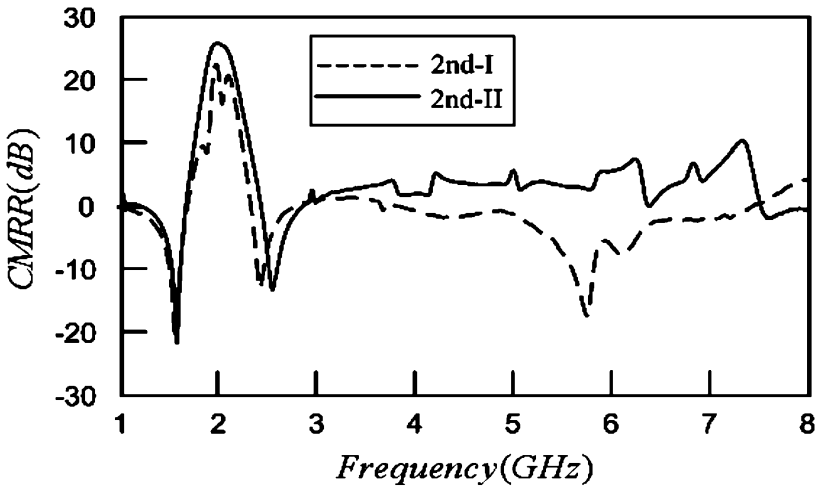

(a)

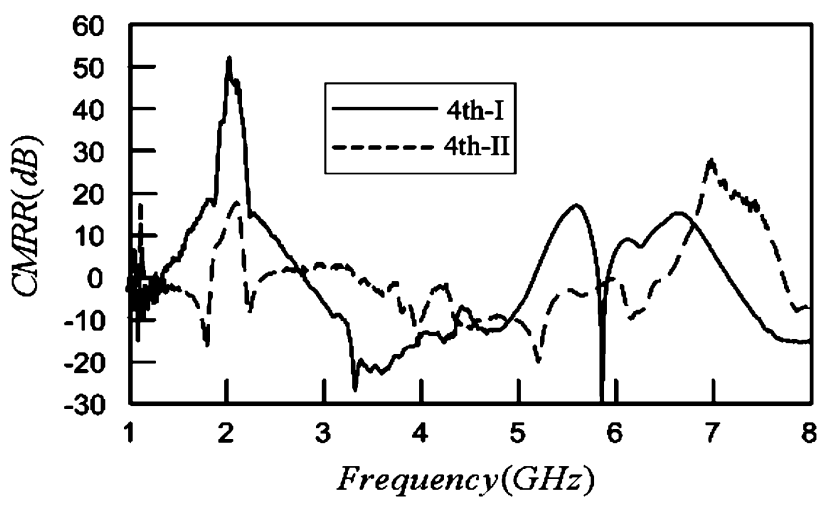

(b)

Fig. 16. Measured CMRR for: (a) second- and (b) fourth-order balanced filters.

common-mode insertion losses $\left|S_{21}^{d d}\right|$ and $\left|S_{21}^{c c}\right|$ may be expressed as

$$
\begin{aligned}
\left|S_{21}^{d d}\right| & =|A+B \angle \Delta \phi| \\
\left|S_{21}^{c c}\right| & =|A-B \angle \Delta \phi|
\end{aligned}
$$

where

$$
\begin{aligned}
A & =\left|S_{21}\right|=\left|S_{2^{\prime} 1^{\prime}}\right| \\
B & =\left|S_{2^{\prime} 1}\right|=\left|S_{21^{\prime}}\right| \\
\Delta \phi & =\left(\angle S_{21}-\angle S_{2^{\prime} 1}\right)-180^{\circ} \\
& =\left(\angle S_{2^{\prime} 1^{\prime}}-\angle S_{21^{\prime}}\right)-180^{\circ}
\end{aligned}
$$

If the balanced circuit were ideal so that

$$
A=B \text { and } \Delta \phi=0^{\circ}
$$

its CMRR would approach infinity, implying that the commonmode signal would completely be suppressed. Thus, in implementing a balanced filter, it is better to nearly meet the balanced conditions (10) so that the undesired signal imbalance $(A \neq B$ and $\Delta \phi \neq 0^{\circ}$ ) may keep to a minimum.

The CMRR provides an important figure-of-merit for a meaningful characterization of balanced circuits. Fig. 16 shows the measured responses of the CMRR for the implemented secondand fourth-order filters. Specifically, the second-order type-II design has a maximum CMRR of $25.7 \mathrm{~dB}$ at $1.97 \mathrm{GHz}$ with all 
TABLE I

Performance Comparison With Previous Studies

\begin{tabular}{|c|c|c|c|c|c|c|}
\hline & $\begin{array}{c}\mathrm{f}_{0} \\
(\mathrm{GHz})\end{array}$ & $\begin{array}{c}\mathrm{FBW} \\
(\%)\end{array}$ & $\begin{array}{c}\mid S_{21}^{d d} \\
(d B)\end{array}$ & $\begin{array}{c}\mathrm{CMRR} \\
(\mathrm{dB})\end{array}$ & $\begin{array}{c}\left|\mathrm{S}_{21}\right|-\mid \mathrm{S}_{2}, \mathrm{l} \\
(\mathrm{dB}) @ \mathrm{f}_{0}\end{array}$ & $\begin{array}{c}\angle \mathrm{S}_{21}-\angle \mathrm{S}_{2,1} \\
(\mathrm{deg}) @ \mathrm{f}_{0}\end{array}$ \\
\hline$[16]$ & 41 & 20 & 4 & $\begin{array}{c}\sim 5 @ \mathrm{f}_{0}, \text { single } \\
\text { element }\end{array}$ & & \\
\hline$[17]$ & 2.15 & 8.5 & 3.36 & $\sim 5 @ \mathrm{f}_{0}$ & & \\
\hline 2nd-I & 2 & 10.5 & 2.9 & $>15$ in passband & 1.972 & 181.8 \\
\hline 2nd-II & 2 & 12 & 2.3 & $>22.5$ in passband & 0.838 & 182.8 \\
\hline 4 th-I & 1.99 & 11 & 3.74 & $>40$ in passband & 0.16 & 179.46 \\
\hline 4th-II & 2.05 & 9.7 & 3.98 & $>12.5$ in passband & 3.807 & 186.51 \\
\hline
\end{tabular}

CMRR values above $22.5 \mathrm{~dB}$ from 1.88 to $2.12 \mathrm{GHz}$. As to the fourth-order type-I filter, it has a maximum CMRR of $52.2 \mathrm{~dB}$ at $2.03 \mathrm{GHz}$ and all CMRR values are above $40 \mathrm{~dB}$ from 1.93 to $2.12 \mathrm{GHz}$, which is almost twice the second-order design.

Four filter circuits realized in this study are summarized and compared with the previous works in Table I. The corresponding values for $\left|S_{21}\right|-\left|S_{2^{\prime} 1}\right|$ and $\angle S_{21}-\angle S_{2^{\prime} 1}$ around $f_{0}^{d}$ are also illustrated in Table I to demonstrate the signal-imbalance phenomena. The CMRR of type-I and type-II second-order designs are roughly 10 and $17.5 \mathrm{~dB}$ higher than that in [17], respectively. This demonstrates that balanced filters with excellent CMRR may be achieved through proper arrangement of the circuit configuration. In addition, the same technique is extended to realize the fourth-order filters for better CMRR. The well-designed fourth-order type-I filter shows an improvement of $35 \mathrm{~dB}$ in CMRR when compared with the one in [17].

\section{CONCLUSIONS}

Four novel balanced filters based on balanced coupled-line sections have been proposed and carefully examined. The adoption of the symmetrical structure and balanced coupled-line sections makes it possible to realize a balanced filter, which gives the desired differential-mode bandpass response, and also minimize the common-mode signal level on the other hand. The cross-coupled effect is also introduced to improve the filter selectivity; however, it enhances the signal imbalance and degrades the common-mode rejection. Specifically, by properly choosing the filter configuration, one may push the commonmode spurs to the higher frequency so that a filter with good selectivity and excellent CMRR may be achieved simultaneously. The CMRR of the well-designed fourth-order type-I balanced filter is above $40 \mathrm{~dB}$ around the differential-mode passband, which is very attractive for balanced topology applications.

\section{REFERENCES}

[1] B. Razavi, Design of Analog CMOS Integrated Circuit. Boston, MA: McGraw-Hill, 2001.

[2] D. Raicu, "Design of planar, single-layer microwave baluns," in IEEE MTT-S Int. Microw. Symp. Dig., 1998, pp. 801-804.

[3] A. M. Pavio and A. Kikel, "A monolithic or hybrid broadband compensated balun," in IEEE MTT-S Int. Microw. Symp. Dig., 1990, pp. 483-486.

[4] K. Nishikawa, I. Toyoda, and T. Tokumitsu, "Compact and broadband three-dimensional MMIC balun," IEEE Trans. Microw. Theory Tech, vol. 47, no. 1, pp. 96-98, Jan. 1999.

[5] S. P. Ojha, G. R. Branner, and B. P. Kumar, "A miniaturized lumped-distributed balun for modern wireless communication systems," in Proc. IEEE Midwest Circuits Syst. Symp., 1996, pp. $1347-1350$.

[6] B. P. Kumar, G. R. Branner, and B. Huang, "Parametric analysis of improved planar balun circuits for wireless microwave and RF applications," in Proc. IEEE Midwest Circuits Syst. Symp., 1998, pp. 474-475.

[7] C. W. Tang and C. Y. Chang, "A semi-lumped balun fabricated by low temperature co-fired ceramic," in IEEE MTT-S Int. Microw. Symp. Dig., 2002, pp. 2201-2204.

[8] Y. C. Leong, K. S. Ang, and C. H. Lee, "A derivation of a class of 3-port baluns from symmetrical 4-port networks," in IEEE MTT-S Int. Microw. Symp. Dig., 2002, pp. 1165-1168.

[9] K. S. Ang, Y. C. Leong, and C. H. Lee, "Analysis and design of miniaturized lumped-distributed impedance-transforming balun," IEEE Trans. Microw. Theory Tech, vol. 51, no. 3, pp. 1009-1017, Mar. 2003.

[10] D. W. Yoo, E. S. Kim, and S. W. Kim, "A balance filter with DC supply for Bluetooth module," in Proc. Eur. Microw. Conf., 2005, pp. $1239-1242$.

[11] M. C. Park, B. H. Lee, and D. S. Park, "A laminated balance filter using LTCC technology," in Proc. Asia-Pacific Microw. Conf., 2005, pp. 4-7.

[12] R. Kravchenko, K. Markov, D. Orlenko, G. Sevskiy, and P. Heide, "Implementation of a miniaturized lumped-distributed balun in balanced filtering for wireless applications," in Proc. Eur. Microw. Conf., 2005, pp. 1303-1306.

[13] L. K. Yeung and K. L. Wu, "An LTCC balanced-to-unbalanced extracted-pole bandpass filter with complex load," IEEE Trans. Microw. Theory Tech, vol. 54, no. 4, pp. 1512-1518, Apr. 2006.

[14] A. Babakhani, X. Guan, A. Komijani, A. Natarajan, and A. Hajimiri, "A $77 \mathrm{GHz} 4$-element phased array receiver with on-chip dipole antenna in silicon," in Int. Solid-State Circuits Conf. Tech. Dig., San Francisco, CA, Feb. 2006, pp. 180-181.

[15] C.-H. Wang, Y. H. Cho, C. S. Lin, H. Wang, C. H. Chen, D. C. Niu, J. Yeha, C. Y. Lee, and J. Chern, "A $60 \mathrm{GHz}$ transmitter with integrated antenna in $0.18 \mu \mathrm{m} \mathrm{SiGe} \mathrm{BiCMOS}$ technology," in Int. Solid-State Circuits Tech. Dig., San Francisco, CA, Feb. 2006, pp. 186-187.

[16] A. Ziroff, M. Nalezinski, and W. Menzel, "A $40 \mathrm{GHz}$ LTCC receiver module using a novel submerged balancing filter structure," in Proc. Radio Wireless Conf., 2003, pp. 151-154.

[17] Y.-S. Lin and C. H. Chen, "Novel balanced microstrip coupled-line bandpass filters," in URSI Int. Electromagn. Theory Symp., 2004, pp. 567-569.

[18] S. B. Cohn, "Parallel-coupled transmission-line-resonator filters," IRE Trans. Microw. Theory Tech., vol. MTT-6, no. 7, pp. 223-231, Apr. 1958.

[19] C.-Y. Chang and T. Itoh, "A modified parallel-coupled filter structure that improves the upper stopband rejection and response symmetry," IEEE Trans. Microw. Theory Tech, vol. 39, no. 2, pp. 310-314, Feb. 1991.

[20] C.-H. Wang, Y.-S. Lin, and C. H. Chen, "Novel inductance-incorporated microstrip coupled-line bandpass filters with two attenuation poles," in IEEE MTT-S Int. Microw. Symp. Dig., 2002, pp. 1979-1982.

[21] Y.-S. Lin, C.-H. Wang, C. H. Wu, and C. H. Chen, "Novel compact parallel-coupled microstrip bandpass filters with lumped-element $K$-inverters," IEEE Trans. Microw. Theory Tech, vol. 53, no. 7, pp. 2324-2328, Jul. 2005.

[22] C.-C. Chen, Y.-R. Chen, and C.-Y. Chang, "Miniaturized microstrip coupled-line bandpass filters using quarter-wave or quasi-quarter-wave resonators," IEEE Trans. Microw. Theory Tech, vol. 51, no. 1, pp. 120-131, Jan. 2003.

[23] D. E. Bockelman and W. R. Eisenstant, "Combined differential and common-mode scattering parameters: Theory and simulation," IEEE Trans. Microw. Theory Tech, vol. 43, no. 7, pp. 1530-1539, Jul. 1995. 


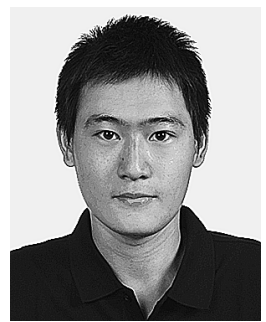

Chung-Hwa Wu (S'06) was born in Tainan, Taiwan, R.O.C., in 1982. He received the B.S. degree in electrical engineering from National Chung Hsing University, Taichung, Taiwan, R.O.C., in 2004, and is currently working toward the Ph.D. degree at the Graduate Institute of Communication Engineering, National Taiwan University, Taipei, Taiwan, R.O.C.

His research interests include the design and analysis of microwave filters and passive circuits.

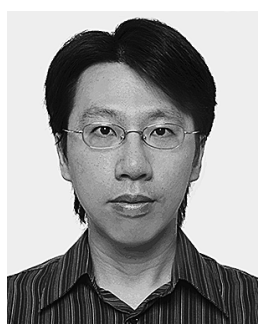

Chi-Hsueh Wang (S'02-M'05) was born in Kaohsiung, Taiwan, R.O.C., in 1976. He received the B.S. degrees in electrical engineering from National Cheng Kung University, Tainan, Taiwan, R.O.C., in 1997, and the Ph.D. degree from National Taiwan University, Taipei, Taiwan, R.O.C., in 2003.

$\mathrm{He}$ is currently a Post-Doctoral Research Fellow with the Graduate Institute of Communication Engineering, National Taiwan University. His research interests include the design and analysis of microwave and millimeter-wave circuits and computational electromagnetics.

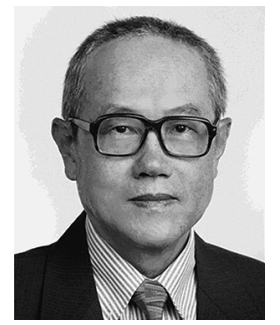

Chun Hsiung Chen (SM'88-F'96) was born in Taipei, Taiwan, R.O.C., on March 7, 1937. He received the B.S.E.E. and Ph.D. degrees in electrical engineering from National Taiwan University, Taipei, Taiwan, R.O.C., in 1960 and 1972, respectively, and the M.S.E.E. degree from National Chiao Tung University, Hsinchu, Taiwan, R.O.C., in 1962.

In 1963, he joined the Faculty of the Department of Electrical Engineering, National Taiwan University, where he is currently a Professor. From August 1982 to July 1985, he was Chairman of the Department of Electrical Engineering, National Taiwan University. From August 1992 to July 1996, he was the Director of the University Computer Center, National Taiwan University. In 1974, he was a Visiting Scholar with the Department of Electrical Engineering and Computer Sciences, University of California at Berkeley. From August 1986 to July 1987, he was a Visiting Professor with the Department of Electrical Engineering, University of Houston, Houston, TX. In 1989, 1990, and 1994, he visited the Microwave Department, Technical University of Munich, Munich, Germany, the Laboratoire d'Optique Electromagnetique, Faculte des Sciences et Techniques de Saint-Jerome, Universite d'Aix-Marseille III, Marseille, France, and the Department of Electrical Engineering, Michigan State University, East Lansing, respectively. His areas of interest include microwave circuits and computational electromagnetics. 